\title{
Controlling and Simulating Soft Robotic Systems: Insights from a Thermodynamic Perspective
}

\author{
Dylan Ross, Markus P. Nemitz, and Adam A. Stokes
}

\begin{abstract}
Soft robots are machines, and like all machines their function is to convert energy from one form into another to perform tasks. One key figure of merit for machines is their efficiency, which is defined as the ratio of taskoriented work out to total energy in. All soft robots convert stored energy (from e.g., batteries, pressurized gas, chemicals) into task-oriented work (picking up objects, locomoting, jumping). These systems are complex hybrids of chemical, mechanical, pneumatic, hydraulic, and electrical components. This complexity makes it difficult to analyze and measure their total efficiency and to identify the sources of energy loss between chemical, electrical, and mechanical domains. As the field of soft robotics matures, the design-flow process will shift from one in which building is central to one in which simulation takes precedence. That is, there is a shift from an empirical experimental methodology toward a well-characterized engineering workflow. At this point, questions such as "For how long will this robot run on a $2000 \mathrm{mAh}$ battery?" will need to be answered, and predictive capabilities will become paramount as designers need to understand: (1) the large-scale deformations inherent to soft robotic systems; and (2) the transduction of energy in these complex, dissipative, systems to enable them to design an efficient and a well-controlled system. In this perspective piece, we discuss one possible predictive approach: a framework that uses port-based modeling. This approach uses bond-graphs and the recently developed port-Hamiltonian theory to provide a step-by-step system for analyzing hybrid, multidomain, soft robotic systems. We discuss how this framework could be applied to controlling and optimizing soft robotic systems for energy efficiency, thereby increasing their utility. An energy-based approach is useful as a domain-free linker in analyzing complex systems; the use of ports promotes a clear distinction between energy conservation and dissipation and facilitates the analysis of efficiency. In addition, the parallels with hardware description languages and object-oriented programming will make it easier for engineers to design, for soft robots, control systems that optimize for efficiency.
\end{abstract}

Keywords: soft robotics, thermodynamics, simulation

\section{Introduction}

Q oft robotic systems are complex hybrids that use $\checkmark$ sources and sinks of chemical, mechanical, pneumatic, hydraulic, and electrical energy. They are machines that have been designed to perform tasks by converting energy from one form (storage) into another (actuation). The fact that they have elements that cross multiple domains, and that they are often made using materials that are capable of very large deformations means that it is difficult for system designers to predict and control their motion and to measure their total efficiency. The control systems for this class of robot are typically open-loop ones, and due to the complexity of analyzing energy transduction from storage to actuation, figures of merit, such as total cost of transport, are very difficult to calculate. $^{1-3}$

Stokes Research Group, Institute for Integrated Micro and Nano Systems, School of Engineering, The University of Edinburgh, Edinburgh, United Kingdom.

(C) Dylan Ross, et al., 2017; Published by Mary Ann Liebert, Inc. This Open Access article is distributed under the terms of the Creative Commons License (http://creativecommons.org/licenses/by/4.0), which permits unrestricted use, distribution, and reproduction in any medium, provided the original work is properly credited. 
In this perspective piece, we suggest that a port-based framework, based on bond-graphs ${ }^{4}$ and the port-Hamiltonian theory, ${ }^{5}$ will be a useful tool for analyzing the thermodynamics underlying hybrid, multi-domain, soft robotic systems. Using this approach will enable designers to identify where energy is lost between source and actuation, across the chemical, mechanical, pneumatic, hydraulic, and electrical domains, and will pave the way for application of energybased control methods in soft robotics.

\section{Design paradigms for hard versus soft robotic systems}

Hard-bodied and soft-bodied robots are designed by using completely different methodologies, as summarized by Figure 1 . One key figure of merit for these machines is efficiency, which is defined as the ratio of task-oriented work out to total energy in. The design methodology and the corresponding ability to predict efficiency are very closely linked.

The design flow for hard robots (Fig. 1a) is one in which simulation is paramount. The system is built by using standardized and well-defined components and rigid links. Testing the physical robot is the last step in the design loop, and it can almost always be replaced with a simulation. The system is controlled by making a predictive model, which is often based on defining the Jacobian of the system and then applying well-established methodologies using inverse kinematics. The efficiency of this class of robotic systems is relatively easy to predict, as it relies on the composition of efficiencies of each of the well-defined components (motors, links, end effectors) in the system. This design flow has parallels with Hardware Description Languages (HDL) and Object-Oriented Programming (OOP)—which hardware and software engineers use to design and to program computer processors. Using these types of paradigms, designers of complex, hard-bodied robotic systems use well-defined and well-characterized blocks.

In stark contrast, the design flow for soft robots (Fig. 1b) is centered on building systems, and it uses an empirical, experimental-science iterative loop. Soft robotic systems are often built by using composite and multi-domain blocks that

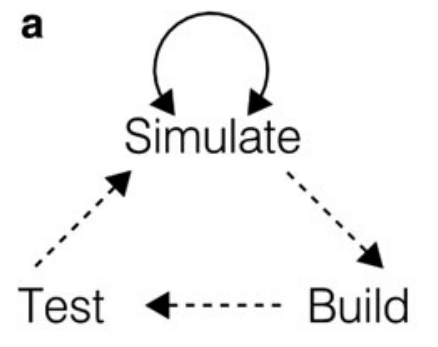

Design Flow for Rigid Robots b

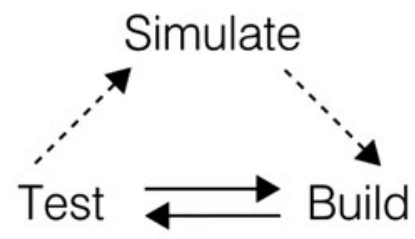

Design Flow for Soft Robots

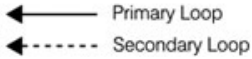

FIG. 1. Overview sketch of the design flows for hard and soft robotic systems, showing: (a) The engineering design flow for rigid robotic systems, in which the simulation loop is paramount and the system is built from well-defined blocks. (b) The experimental-science design flow for soft robotic systems in which the building loop is paramount as the system is built from poorly characterized blocks. are not completely characterized. The system control is often an open-loop one, and locomotive actuation sequences are experimentally determined. Significant efforts toward simulating soft material systems has been made by Cotin and Lipson, resulting in programs such as $\mathrm{SOFA}^{6}$ and $\mathrm{VoxCAD}^{7-9}$, respectively. Testing the physical device is the key iteration in the design loop and, due to the complexity of simulating large deformations, and their strong dependence on interaction with the environment, this loop cannot (yet) be completely replaced by simulation.

This design flow has parallels with early microprocessor designs that were laid out by hand in an empirical, revisionist, and iterative process. All soft robots convert stored energy (from electrochemical cells, ${ }^{10-13}$ pressurized gas, ${ }^{14-17}$ energy-dense chemicals ${ }^{3,18-20}$ ) into task-oriented work (grasping and lifting, ${ }^{21-23}$ locomoting, ${ }^{10,11,16,17,24,25}$ jumping, ${ }^{19,20}$ swimming $\left.{ }^{13}\right)$. In order for system designers to predict how long such a system will run on a given amount of stored energy, they need to understand and model the forms of energy storage and dissipation; only then will they be able to control these systems efficiently.

These types of questions, about efficiency, longevity, and recharging, are common to all sub-fields of mobile robotics, but they are particularly interesting when asked about those robots that: (1) are bioinspired, ${ }^{3}$ as they raise questions about how living systems store and recover energy, and insights into how these mechanisms could be improved; (2) use direct, chemical to mechanical, actuation, ${ }^{19,20}$ as the multi-domain physics is complex; or (3) interact synergistically with biology, for example, a human body, ${ }^{26}$ as the coupling between living and non-living components presents great challenges in both control and safety.

The robotics community is starting to make strides toward developing robots that approach the minimum costs of transport (MCoT) for animals, as defined by Tucker. ${ }^{1}$ One characteristic that is common to all robots, soft or otherwise, is the conversion of energy from one or more storage elements to one or more dissipative elements. These dissipative elements include those that perform task-oriented work and those that dissipate heat-resulting in the irreversible loss of energy to the environment.

Robotic systems can, therefore, be analyzed in terms of fundamental quantities: energy, work, and heat. The MIT cheetah robot, for example, is a robot that has been designed with energy in mind. The creators of this robot-Seok et al.-identify three main sources of energy loss during locomotion: (1) heat losses from the actuators; (2) friction losses in transmission; and (3) interaction losses caused by the interface between the system and the environment. To reduce these sources of energy dissipation, the MIT cheetah system contains regenerative electronic systems, high torquedensity motors, low-loss transmission, and low leg inertia. ${ }^{27}$ Soft systems hold particular promise for decreasing the MCoT in locomoting systems, as they contain structural and actuating elements that are capable of storing and returning energy. Despite this possibility, no soft systems have yet been developed that come close to the low MCoT of the MIT cheetah.

To use elastic structural and actuating elements the most effectively, designers of soft systems will need to understand two grand challenges: (1) Characterizing soft robots in terms of energy transformation, calculating energetic "figures of 
merit," and identifying sources of energy loss; (2) analyzing, modeling, and simulating the whole-body mechanics and dynamics of large-scale deformations in soft robotic systems.

In this article, we offer our perspective on the first of these two grand challenges in soft robotic systems: how to characterize complex, multi-domain hybrid systems. We suggest that a modeling, analysis, and control framework built on bond-graphs and the port-Hamiltonian theory will be of great utility in the future of soft robotics. The framework also provides a link to the second grand challenge, which we do not tackle here. Bond-graphs and the port-Hamiltonian theory describe the flow of energy through a system. They are tools for understanding the thermodynamics and for predicting the dynamic behavior of complex systems.

\section{The role of thermodynamics in robot design-energy: storage, transduction, and dissipation}

Thermodynamics is often overlooked when designing tethered, factory-based, robotic systems, as it is a secondary consideration to the task in hand-rapid, precise manipulation in a structured, well-defined, and people-free environment. In contrast, when designing field robotics-where the task is reconnaissance or transportation-analysis and predictions for storage, transduction, and dissipation of the energy within the system are paramount.

There are well-established modeling approaches that focus on energy. For instance, Hamiltonian mechanics is a reformulation of classical Newtonian mechanics that takes as its starting point the Hamiltonian-a quantity that generally corresponds to the total energy contained in a mechanical system. From this single quantity, it is possible to derive differential equations that govern the motion of the system. Although initially applied only to discrete (lumpedparameter) systems, the Hamiltonian field theory has generalized the concepts of Hamiltonian mechanics to continuum systems. More recently, these ideas have been extended by the port-Hamiltonian theory, ${ }^{5}$ which generalizes Hamiltonian mechanics further to the case of multi-domain, dissipative, and mixed discrete-continuum systems with inputs and outputs. We believe that this theoretical framework provides an ideal tool for the analysis of soft robotic systems.

In two of our previous papers - on hybrid hard and soft robots, ${ }^{11}$ and on using explosions to power soft robots ${ }^{19}$-we began to introduce the idea of analyzing the efficiency and capabilities of these robots by discussing what was known about the transduction of energy in each system. In this article, we use these systems to illustrate the application of a generalized framework for describing energy flow and dynamics for these types of robots. Once expressions for the total energy, power transfer, and dissipation in a robotic system have been formulated by using this approach, they can be used for control and to optimize for efficiency. In the Supplementary Data (Supplementary Data are available online at www.liebertpub.com/soro), we work through two examples that relate to the two systems shown in Figure 3: a rigid link robotic arm and a soft, continuously deformable octopus tentacle. In the Supplementary Data (Figs. S1-S5), we use the same energy-based analytical tools to derive the equations of motion for each of these two systems.

\section{Modeling of complex systems-a brief introduction to bond-graphs}

Bond-graphs. In the $1950 \mathrm{~s}$ at MIT, Henry Paynter developed pictorial representations of interacting energetic elements, bond-graphs, as a way of modeling complex systems. ${ }^{4}$ These graphs are a way of representing the flow of power in systems, and they allow designers to test their assumptions and to draw relationships between interacting elements across multiple domains. Bond-graph theory centers on the concept of ports that connect effort and flow variables, which together carry power. Table 1 shows some flow and effort variables for multiple domains.

Table 1. Variables Used in Bond-Graphs and Port-Hamiltonian Theory That Describe the Flow and Effort That Together Carry Power Transfer Between a Range of Domains, Including Thermal, Mechanical, Pneumatic, Chemical, Electrical, and Magnetic

\begin{tabular}{|c|c|c|c|c|}
\hline & Effort, $e$ & Flow, $f$ & $\begin{array}{l}\text { Generalized } \\
\text { Momentum, } \mathrm{p} \\
p=\int \mathrm{e} \mathrm{dt}\end{array}$ & $\begin{array}{l}\text { Generalized } \\
\text { Displacement, } \mathrm{q} \\
\mathrm{q}=\int \mathrm{fdt}\end{array}$ \\
\hline Electrical & Voltage, V [V] & Current, I [A] & $\begin{array}{l}\text { Magnetic Flux } \\
\text { Linkage, } \varphi[\mathrm{Wb}]\end{array}$ & Charge, $\mathrm{q}[\mathrm{C}]$ \\
\hline Magnetic & $\begin{array}{l}\text { Magnetomotive } \\
\text { Force, } \mathrm{e}_{\mathrm{m}}[\mathrm{A} \cdot \text { turns }]\end{array}$ & $\begin{array}{l}\text { Magnetic Flux Rate, } \\
\varphi[\mathrm{Wb} / \mathrm{sec}]\end{array}$ & Not defined & $\begin{array}{l}\text { Magnetic Flux, } \\
\qquad \varphi[\mathrm{Wb}]\end{array}$ \\
\hline $\begin{array}{l}\text { Mechanical } \\
\text { (Translational) }\end{array}$ & Force, $\mathrm{F}[\mathrm{N}]$ & Velocity, v [m/s] & $\begin{array}{l}\text { Momentum, } p=\int \\
\quad \mathrm{F} \text { dt }[\mathrm{N} \cdot \mathrm{sec}]\end{array}$ & $\begin{array}{l}\text { Displacement, } \\
\text { r [m] }\end{array}$ \\
\hline $\begin{array}{l}\text { Mechanical } \\
\text { (Rotational) }\end{array}$ & Torque, $\tau[\mathrm{N} \cdot \mathrm{m}]$ & $\begin{array}{l}\text { Angular Velocity, } \\
\omega[\mathrm{rad} / \mathrm{sec}]\end{array}$ & $\begin{array}{l}\text { Angular momentum, } \\
\quad \mathrm{b}=\varphi=\int \tau \mathrm{dt} \\
{[\mathrm{N} \cdot \mathrm{m} \cdot \mathrm{sec}]}\end{array}$ & $\begin{array}{l}\text { Angular } \\
\text { displacement, } \\
\theta \text { [rad] }\end{array}$ \\
\hline $\begin{array}{l}\text { Hydraulic/ } \\
\text { Pneumatic }\end{array}$ & Pressure, $\mathrm{P}\left[\mathrm{N} / \mathrm{m}^{2}\right]$ & $\begin{array}{l}\text { Volumetric Flow, } \\
\mathrm{dQ} / \mathrm{dt}\left[\mathrm{m}^{3} / \mathrm{sec}\right]\end{array}$ & $\begin{array}{l}\text { Momentum of a flow } \\
\text { tube, } \Gamma=\int_{\mathrm{S}} \mathrm{P} \\
\mathrm{dt}\left[\mathrm{N} / \mathrm{m}^{2} \mathrm{~s}\right]\end{array}$ & Volume, $\mathrm{Q}\left[\mathrm{m}^{3}\right]$ \\
\hline Thermal & Temperature, $\mathrm{T}[\mathrm{K}]$ & Entropy Flow, dS/dt [W/K] & Not defined & Entropy, S [J/K] \\
\hline Thermal & Pressure, $\mathrm{P}\left[\mathrm{N} / \mathrm{m}^{2}\right]$ & $\begin{array}{l}\text { Volume Change, } \\
\mathrm{dV} / \mathrm{dt}\left[\mathrm{m}^{3} / \mathrm{sec}\right]\end{array}$ & Not defined & Volume, $\mathrm{V}\left[\mathrm{m}^{3}\right]$ \\
\hline Chemical & $\begin{array}{l}\text { Chemical Potential, } \\
\mu[\mathrm{J} / \mathrm{mol}]\end{array}$ & $\begin{array}{l}\text { Molar Flow, } \\
\text { dN/dt [mole/sec] }\end{array}$ & Not defined & $\begin{array}{l}\text { Number of moles, } \\
\quad \mathrm{N}[\mathrm{mol}]\end{array}$ \\
\hline Chemical & Enthalpy, H [J/kg] & Mass Flow, dm/dt $[\mathrm{g} / \mathrm{sec}]$ & Not defined & Mass, m [g] \\
\hline
\end{tabular}




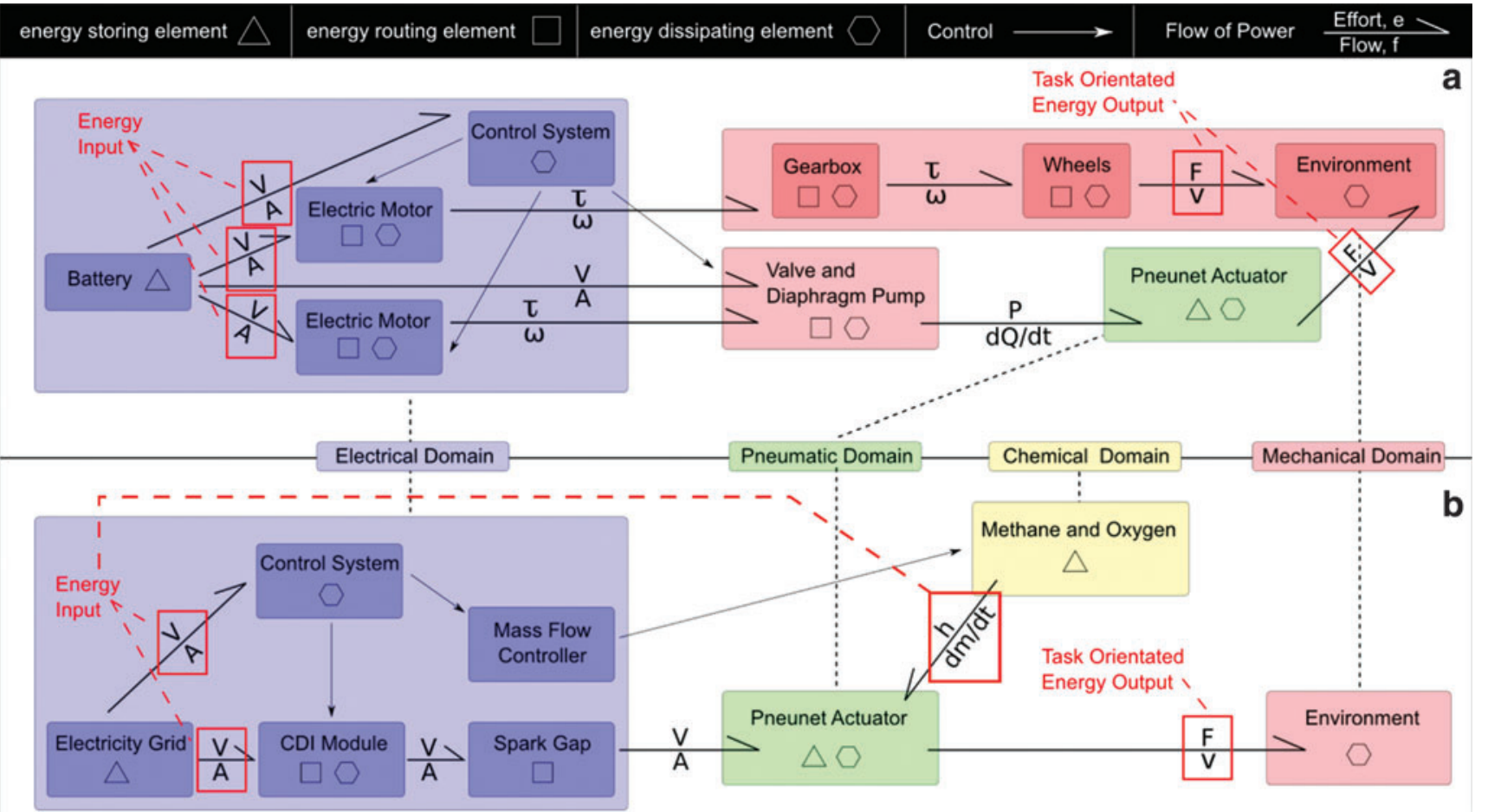

FIG. 2. Word bond-graph diagrams showing: the control; the sources; the sinks; and the flow of energy between mechanical, electrical, pneumatic, and chemical domains in: (a) A hybrid combining hard and soft robots, ${ }^{2}$ and (b) A soft robot powered by explosions. This type of diagram can be used to identify assumptions about the model, and to break down complex systems based on the flow and dissipation of energy, from storage through transmission, and to task-oriented work performed. The effort and flow variables used are described in Table 1. Color images available online at www.liebertpub.com/soro

In the bond-graph diagrams, "ports" between system blocks are connected with half-arrow bonds showing the usual direction of power flow. The properties of a port are the most easily understood by considering the basic electrical circuit theory, in which the flow variable is current (I), and the effort variable is voltage (V). Loss would be characterized by dissipative elements representing Ohmic $\left(\mathrm{I}^{2} \mathrm{R}\right)$ heating. The ideas, however, have direct analogs across multiple physical domains; see Table 1 for examples.

\section{Word bond-graph analysis of two soft robotic systems}

Bond-graph analysis of a hybrid soft robotic system. Figure 2a (derived from Supplementary Fig. S6a) shows a word bond-graph multi-domain block diagram for a hybrid soft robotic system. In this system, energy is derived from the electricity grid and task-oriented energy is dissipated by the interaction of the wheels and the pneunets with the environment. The global efficiency of this system can be calculated as the ratio between the sum of the electrical input energy and the mechanical output from the reaction of the wheels and the pneunet with the environment. This bond-graph analysis allows us to identify what we need to know to simulate this system. For example, we do not know how to simulate the link between the power into a pneunet actuator (from pressure and volumetric flow rate) and the power out (force and velocity). This analysis also reveals what type of sensors we would need to deploy in the system to monitor the flow and dissipation of energy. If we consider Table 1 , we can see that there are a variety of parameters that designers would not routinely
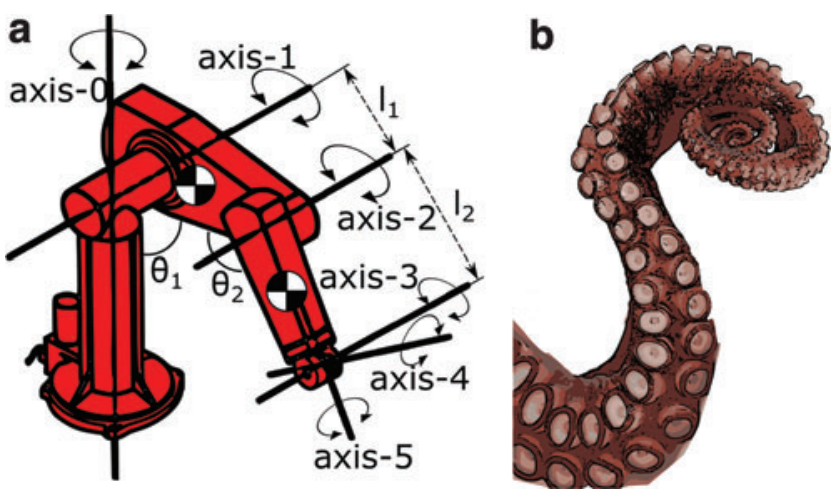

FIG. 3. Clearly, there are significantly different challenges in modeling the kinematics and energetics of: (a) A simple rigid bodied system (PUMA robot), and (b) A complex softbodied system (Octopus). These two systems can perform the same task-gripping-but each uses completely different mechanics, control systems, friction models, and so on. Control paradigms that have been developed for rigid bodied systems such as the PUMA robot (e.g., deriving the Jacobian and computing the inverse kinematics) have little or no relevance to soft-bodied systems that have more characteristics in common with the octopus arm. The taskoriented work performed by each system-such as gripping and lifting an object-is, however, the same. Color images available online at www.liebertpub.com/soro 
include in a non-energy-based system controller. This type of insight is critical in the design of energy optimal systems.

Bond-graph analysis of a soft robot powered by explosions. Figure 2b (derived from Supplementary Fig. S6b) shows a word bond-graph multi-domain block diagram for a soft robot that is powered by explosions. The global efficiency of this system can be calculated as the ratio between the sum of the electrical and chemical input energy and the mechanical output from the reaction of the pneunet with the environment. After this process, as described earlier, we find that we do not know how to simulate the link between power into an exploding soft actuator (spark voltage and current, and enthalpy and mass flow rate) and the power out (force and velocity). We can measure the energy in (chemical potential, mass flow) and the useful work out (potential energy developed in jumping); therefore, we have a measure of the energy that is dissipated in the system, but predicting or simulating this block is complex.

\section{From bond-graph to dynamics: the port-Hamiltonian approach}

In the previous two sections, we discussed how the construction of a word bond-graph can help in identifying sources of energy loss and in evaluating the efficiency of a system. Bond-graphs can also be powerful tools for taking a high-level description of a system to the point of mathematical analysis and simulation. To begin this process, we must detail the energetic transformations occurring within each block of an abstract word bond-graph. This is a recursive process in which blocks are replaced with more detailed bond-graphs. The aim is to arrive at a system description in which each block represents a fundamental energy storage, transport, transduction, or dissipation element with a welldefined constitutive relationship between its power-conjugate flow and effort variables. Several excellent examples of how to reduce word bond-graphs to a minimal set of fundamental elements covering many physical domains can be found in the literature. $^{28}$

Having produced a detailed bond-graph describing a system, it is possible to apply the tools of the port-Hamiltonian theory to derive the differential-algebraic equations governing the dynamics of the system. ${ }^{28}$ This theory extends energy-conservative Hamiltonian mechanics to the case of multi-domain, dissipative systems with inputs and outputs. The central quantity in this theory is the Hamiltonian-which generally represents the total energy stored within a system and can be constructed by consideration of the energy storage elements in a bond-graph. In Hamiltonian mechanics, this quantity is usually a sum of kinetic and mechanical potential (e.g., gravitational, elastic) energy. In port-Hamiltonian theory, it may equally contain terms for chemical energy or energy associated with electric and magnetic fields. These elements are interconnected mathematically via a powerconservative Dirac structure, whose form may be derived from the energy transport and transduction elements appearing in a detailed bond-graph. This structure not only allows the individual stores of energy represented in the Hamiltonian to interact but also connects them to dissipative elements that irreversibly remove energy from the system as heat. The fundamentals of energy storage, transport, trans- duction, and dissipation are common to both hard and soft robotics. Thus, the tools of the port-Hamiltonian theory can be applied equally well to either. In the case of an entirely lumped-parameter system, for example a traditional robot with discrete electronics and rigid mechanical elements, the port-Hamiltonian dynamics equations take the following form:

$$
x^{\prime}(t)=\left[J\left(x^{\prime}\right)-R(x)\right] \partial_{x} H(x) \text { where } \partial_{x}=\frac{\partial}{\partial x}
$$

In a completely distributed-parameter system, they take the form:

$$
\begin{aligned}
x^{\prime}(X, t) & =\left[J\left(x^{\prime}\right)-R(x)\right] \partial_{x} H(x) \text { where } \\
\partial_{x} & =\frac{\partial}{\partial x}-\frac{\partial}{\partial t}\left[\frac{\partial}{\partial_{t} x}\right]-\nabla \cdot\left[\frac{\partial}{\partial_{X} x}\right]
\end{aligned}
$$

In the lumped-parameter case, $x$ is a vector of state variables that are derived from the bond-graph's flow and effort variables, and $H$ is the Hamiltonian. In the distributedparameter case, $x$ is now a vector of field variables (e.g., the mechanical displacement and momentum fields for a deformable soft body) that depend on spatial coordinates $X$, and $H$ is the Hamiltonian density-usually the energy density. In both cases, $J$ is a skew-symmetric map representing the power-conservative Dirac structure of the system, and $R$ is a perturbation to $J$ that allows for dissipation. Combinations of lumped- and distributed-parameter elements, a situation commonly encountered in soft robotics, are equally well treated by the port-Hamiltonian approach.

The procedure for deriving these equations of motion from a given bond-graph is systematized, and it can even be carried out algorithmically. ${ }^{16}$ This means that the roboticist is able to focus on an intuitive, pictorial representation (the bondgraph) of the system being designed. Furthermore, once a detailed bond-graph has been constructed for a given subsystem, it can be reused several times. So long as constitutive equations for the elements in the system can be provided, the difficult work of deriving the system's dynamics is taken care of.

The port-Hamiltonian theory can also be put to good use in developing controllers for complex systems. For instance, there has been much success in using passivity or energyshaping control to alter the static and dynamic behavior of port-Hamiltonian systems. ${ }^{28,29}$ Given the generality and strong physical basis of this theoretical approach, many of the control techniques can be readily applied to soft, continuum systems. ${ }^{30}$

\section{Gaining insights by using an energy-based analytical framework}

The step-by-step framework we are proposing allows system designers to start with a complex system and to move toward an energy-based system controller; this approach is composed of six steps: (1) Writing the word bond-graph; (2) refining to a detailed bond-graph; (3) minimizing the bondgraph; (4) developing the port-Hamiltonian and Dirac structures; (5) deriving the equations of motion; and finally (6) coding the system controller.

A limitation, which we identified by constructing both of the bond-graph analyses that we presented earlier in this 
article, is that the block representing the pneumatic actuator has not yet been represented mathematically. This abstract block represents a complex interplay of elements: 3D viscoelastic polymers are subjected to surface pressures from a compressible gas, whereas dynamic reaction forces appear due to an interaction of the whole network with a surface. On breaking this system into more basic energetic components, we realize that we do not know how to model the storage and loss of energy in the viscoelastic polymer. The construction of bond-graphs for these systems and progression toward simulation has highlighted exactly what empirical work is critical for the analysis of the system; we need to develop explicit constitutive relations for the viscoelastic polymer.

Note, however, that this need not stop us from proceeding to analysis, simulation, and control. If we approximate this constitutive relation - for instance, by assuming infinitesimal strains and a linear material response ${ }^{31}$ — we can begin by using the tools of the port-Hamiltonian theory to derive the dynamics for the entire, multi-domain system, and thus begin computational analysis. All models are based on theories; they require us to explicitly state our assumptions, and they allow us to test our understanding. The approach of building bond-graphs and moving toward simulation by making successive approximations can help us find out where we should focus our future efforts on theoretical and empirical work.

\section{Conclusions}

\section{Implications for future robotic systems}

In this perspective piece, we have discussed how the use of bond-graphs and the port-Hamiltonian theory generalizes domain-specific knowledge and allows engineers to analyze complex and hybrid systems. We hope to popularize a mature framework for addressing energetic concerns in soft robotics, and we expect that it may also be used, systematically, to derive equations governing coupled, multidomain dynamics. Using the insights gained from this type of holistic system overview - and one based on energyengineers will be able to use elastic, energy-storing, structural, and actuating elements the most effectively in future soft robotic systems. Bond-graphs are clearly a useful tool for conceptualizing a system at various levels of abstraction. The application of the port-Hamiltonian theory requires us to make quantitative modeling decisions, and to identify those areas in which idealization or empirical analysis is the most needed, the methods we have discussed here offer a significant step toward incorporating mathematical analysis, simulation, and control into the design flow of complex soft robotic systems.

\section{Acknowledgments}

The authors thank the members of The Stokes Research Group at The University of Edinburgh, The Robosoft Community, and the MINIMAL consortium for useful conversations and comments on this article. This study was supported by EPSRC via the Robotarium Capital Equipment and CDT Capital Equipment Grants (EP/L016834/1), and by the FP7 Robosoft CA and MINIMAL grants. Markus Nemitz gratefully acknowledges support from the CDT in Integrative Sensing and Measurement (EP/L016753/1).

\section{Author Disclosure Statements}

No competing financial interests exist.

\section{References}

1. Tucker VA. The Energetic Cost of Moving About. Am Sci 1975;63:413-419.

2. Roberts TJ, Kram R, Weyand PG. Energetics of bipedal running. I. Metabolic cost of generating force. J Exp Biol 1998; 201(Pt 19):2745-2751.

3. Messner P, Paik J, Shepherd R, Kim S. Energy for biomimetic robots: Challenges and solutions. Soft Robot 2014;2: 106-109.

4. Paynter HM. An Epistemic Prehistory of Bond Graphs (Bond Graphs for Engineers) Breedveld PC, DauphinTanguy G (Eds). Amsterdam: Elesvier, 1992.

5. Bedford A. Hamilton's Principle in Continuum Mechanics. Boston-London-Melbourne: Pitman Publishing, 1985.

6. Duriez C, Allard J, Faure F, Bensoussan P-J, Delingette H, Cotin S. EP4A: Software and computer based simulator research: Development and outlook SOFA-An open source framework for medical simulation. Simul Healthc 2007;2:284-285.

7. Hiller J, Lipson H. Dynamic simulation of soft multimaterial 3d-printed objects. Soft Robot 2014;1:88-101.

8. Hiller J, Lipson H. Dynamic simulation of soft heterogeneous objects. arXiv preprint arXiv:1212.2845. 2012.

9. Lipson H. Challenges and opportunities for design, simulation, and fabrication of soft robots. Soft Robot 2014;1: 21-27.

10. Lin H-T, Leisk G, Trimmer B. GoQBot: A CaterpillarInspired Soft-Bodied Rolling Robot. Bioinspir Biomim 2011;6:026007.

11. Stokes A, Shepherd R, Morin S, Ilievski F, Whitesides G. A hybrid combining hard and soft robots. Soft Robot 2013; 1:70-74.

12. Marchese AD, Onal CD, Rus D. Soft robot actuators using energy-efficient valves controlled by electropermanent magnets. San Francisco, CA: IEEE/RSJ International Conference on Intelligent Robots and Systems (IROS), 2011.

13. Marchese AD, Onal CD, Rus D. Autonomous Soft Robotic Fish Capable of Escape Maneuvers Using Fluidic Elastomer Actuators. Soft Robot 2014;1:75-87.

14. Gupta U, Shim J, Bertoldi K, Walsh CJ. Pneumatic networks for soft robotics that actuate rapidly. Adv Funct Mater 2014;24:2163-2170.

15. Pigula FA, Mooney DJ, Bertoldi K, Walsh CJ. A bioinspired soft actuated material. Adv Mater 2014;26:12001206.

16. Shepherd RF, Ilievski F, Choi W. Multigait Soft Robot. Proc Natl Acad Sci U S A 2011;108:20400-20403.

17. Tolley M, Shepherd R, Mosadegh B, Galloway K, Wehner M, Karpelson M, et al. A Resilient, Untethered Soft Robot. Soft Robot 2014;1:213-223.

18. Loepfe M, Schumacher CM, Lustenberger UB. An Untethered, Jumping Roly-Poly Soft Robot Driven by Combustion. Soft Robot 2015;2:33-41.

19. Shepherd RF, Stokes AA, Freake J, Barber J. Using explosions to power a soft robot. Angew Chem Int Ed Engl 2013; 52:2892-2896.

20. Bartlett N, Tolley M, Overvelde J, Weaver J, Mosadegh B, Bertoldi K, et al. A 3D-printed, functionally graded soft robot powered by combustion. Science 2015;349:161-165. 
21. Ilievski F, Mazzeo AD, Shepherd RF, Chen X, Whitesides GM. Soft robotics for chemists. Angew Chem Int Ed Engl 2011;50:1890-1895.

22. Laschi C, Cianchetti M, Mazzolai B, Margheri L. Soft robot arm inspired by the octopus. Adv Robot 2012;26: 709-727.

23. Calisti M, Giorelli M, Levy G, Mazzolai B, Hochner B, Laschi C. An octopus-bioinspired solution to movement and manipulation for soft robots. Bioinspir Biomim 2011;6: 036002.

24. Wei T, Stokes AA, Webb B. A Soft Pneumatic Maggot Robot. 5th International Conference on Biomimetic and Biohybrid Systems 2016 Jul 19. Edinburgh: Springer International Publishing, 2016.

25. Boxerbaum AS, Chiel HJ, Quinn RD. Continuous wave peristaltic motion in a robot. Int J Robot Res 2012;31:302318.

26. Wehner M, Tolley MT, Mengüç Y, Park YL. Pneumatic energy sources for autonomous and wearable soft robotics. Soft Robot 2014;1:263-274.

27. Seok S, Wang A, Chuah MY, Hyun DJ, Lee J, Otten DM. design principles for energy-efficient legged locomotion and implementation on the MIT Cheetah Robot. IEEE/ ASME Transact Mech 2015;20:1117-1129.
28. Duindam V, Macchelli A, Stramigioli S, Bruyninckx H. Modeling and Control of Complex Systems (The PortHamiltonian Approach). New York: Springer-Verlag 2009.

29. Ortega R, Schaft V, Mareels I, Maschke B. Putting energy back in control. IEEE Control Syst Mag 2001;21:18-33.

30. Siuka A, Schöberl M, Schlacher K. Port-Hamiltonian Modelling and Energy-Based Control of the Timoshenko Beam. Acta Mech 2011;222:69-89.

31. Landau LD, Lifshitz EM, Sykes JB, Bell, JS, Alverson AC. Mechanics. Phys Today 1962;15:48.

Address correspondence to: Adam A. Stokes Stokes Research Group Institute for Integrated Micro and Nano Systems School of Engineering

The University of Edinburgh The King's Buildings Edinburgh EH9 $3 \mathrm{LJ}$ United Kingdom

E-mail: a.a.stokes@ed.ac.uk 